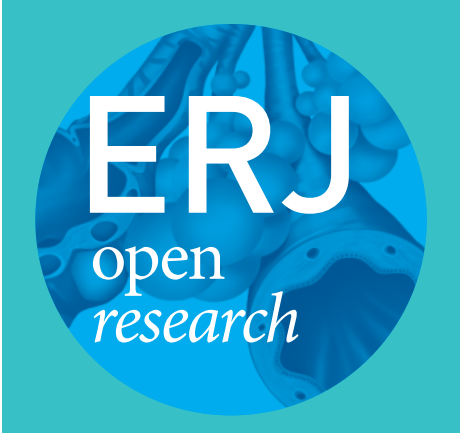

\title{
Longitudinal monitoring of sinonasal and oral bacterial reservoirs to prevent chronic lung infection in people with cystic fibrosis
}

\author{
Rebeca Passarelli Mantovani ${ }^{1,3}$, Angela Sandri, ${ }^{1,3}$, Marzia Boaretti ${ }^{1}$, \\ Gloria Burlacchini ${ }^{1}$, Veronica Li Vigni ${ }^{1}$, Mattia Scarazzai ${ }^{1}$, Paola Melotti ${ }^{2}$, \\ Caterina Signoretto ${ }^{1,4}$ and Maria M. Lleo ${ }^{1,4}$
}

Affiliations: ${ }^{1}$ Dept of Diagnostics and Public Health, Microbiology Section, University of Verona, Verona, Italy. ${ }^{2}$ Cystic Fibrosis Centre, Azienda Ospedaliera Universitaria Integrata (AOUI) of Verona, Verona, Italy. ${ }^{3}$ Co-first authors. ${ }^{4} \mathrm{Co}$-senior authors.

Correspondence: Caterina Signoretto, Dept of Diagnostics and Public Health, Microbiology Section, Strada Le Grazie 8, 37134 Verona, Italy. E-mail: caterina.signorettodunivr.it

\section{ABSTRACT}

Background: Paranasal sinuses act as bacterial reservoirs and contribute to transmitting bacteria to the lower airway of patients with cystic fibrosis (CF). Also, passage of bacteria from the oral cavity to the lungs may occur.

Methods: We evaluated the presence of Pseudomonas aeruginosa, Staphylococcus aureus, Stenotrophomonas maltophilia, Achromobacter xylosoxidans and Serratia marcescens in sputum and nasal lavage of 59 patients with $\mathrm{CF}$, and also collected saliva and used toothbrushes from 38 of them. We assessed the clonal identity of the strains isolated from the different samples by pulsed-field gel electrophoresis.

Results: About $80 \%$ of the patients were positive for at least one of the bacterial species examined in nasal lavage and sputum. Among the subjects with positive sputum, 74\% presented the same species in the nasal lavage and saliva, and $26 \%$ on their toothbrush. S. aureus was the most abundant species in all samples. Clonal identity $(\geqslant 80 \%$ similarity) of the strains isolated among the different samples from each patient was confirmed in almost all cases. Longitudinal observation helped to identify five patients who were colonised in the lower airways after an initial period of nasal or oral colonisation.

Conclusion: Nasal and oral sites act as bacterial reservoirs, favouring the transmission of potentially pathogenic microorganisms to the lower airway. The lack of eradication from these sites might undermine the antibiotic therapy applied to treat the lung infection, allowing the persistence of the bacteria within the patient if colonisation of these sites is not assessed, and no specific therapy is performed.

@ERSpublications

Nasal and oral sites act as bacterial reservoirs favouring the transmission of potentially pathogenic microorganisms to the lower airways of patients with cystic fibrosis https://bit.ly/ 2TMomjk

Cite this article as: Passarelli Mantovani R, Sandri A, Boaretti M, et al. Longitudinal monitoring of sinonasal and oral bacterial reservoirs to prevent chronic lung infection in people with cystic fibrosis. ERJ Open Res 2020; 6: 00115-2020 [https://doi.org/10.1183/23120541.00115-2020].

This article has supplementary material available from openres.ersjournals.com

Received: 4 March 2020 | Accepted after revision: 21 May 2020

Copyright $\odot$ ERS 2020. This article is open access and distributed under the terms of the Creative Commons Attribution Non-Commercial Licence 4.0. 


\section{Introduction}

Chronic pulmonary infection caused by opportunistic bacteria is the major cause of morbidity and mortality in cystic fibrosis (CF) [1]. Staphylococcus aureus is the first bacterial species infecting CF airways during childhood, while Pseudomonas aeruginosa later chronically colonises up to $80 \%$ of adults with CF. $P$. aeruginosa eradication is possible during early colonisation as long as colonies do not evolve into mucoid phenotypes, but when the infection becomes chronic there are few opportunities for a successful therapeutic intervention [2]. It is then necessary to identify and treat all the bacterial reservoirs and those factors promoting the passage from acute to chronic infection to avoid or delay persistent lung colonisation.

It has been shown that the paranasal sinuses are a bacterial reservoir for pulmonary infections in patients with CF [3]. In particular, chronic rhinosinusitis and nasal polyposis are hallmarks in the CF population. Characterised by bacteria persisting in the sinuses for years, they can be a focus for initial lung colonisation and maintenance of the infection. Moreover, changes in computed tomography exams indicate that sinonasal diseases and symptoms appear soon after birth, which suggest a careful examination of the upper airways in children with CF [4,5]. Performing nasal and paranasal endoscopy, SAKANO et al. [6] also showed that only 1 of 50 subjects enrolled in their study did not present nasal and paranasal sinuses affections. Interestingly, the studies addressing the nasal sites as bacterial reservoirs have shown that $P$. aeruginosa strains isolated from the nasal sinus and the sputum of the same patient have a common genomic profile, indicating that they belong to the same clone circulating within the airways [3, 7, 8]. Moreover, it is estimated that patients with $P$. aeruginosa and $S$. aureus colonisation in the upper airway (UAW) have higher probability (4000 and 25 times, respectively) of lower airway (LAW) colonisation with the same species [9]. Additionally, the paranasal sinuses are not adequately reached by antibiotics applied by conventional inhalation or intravenous treatment, therefore facilitating bacterial persistence [10]. On the other hand, other possible bacterial reservoirs could be considered. As an example, the passage of bacteria from the oral cavity to the lungs may occur by aspiration of pathogens then released in the saliva or may be facilitated by the passage of medical devices such as bronchoscopes and endotracheal tubes [11]. For this reason, we have considered in this study the oral cavity and an object used daily for oral treatment such as the toothbrush as possible reservoirs.

Most studies on bacterial reservoirs in CF have been conducted in a small number of subjects, while there is a need to investigate a higher number of individuals with $\mathrm{CF}$ to assess whether the monitoring and eradication of the bacterial flora in the reservoirs would be a good practice to be introduced in the clinical field to avoid or delay chronic lung infection. A clinical study conducted by MAINZ et al. [12] on over 180 patients compared the microbial flora in the UAW and LAW and assessed the genetic relatedness of $S$. aureus and $P$. aeruginosa strains, the most frequent species inhabiting the UAW and LAW of patients with $\mathrm{CF}$, collected in the two different environments. However, on the basis of the increasingly frequent detection of emergent pathogens colonising the CF lung, it would be interesting to evaluate whether other bacterial species could also be present in the paranasal sinus and oral cavity and constitute a reservoir for colonisation of the lung. To this purpose, we have monitored the presence of $P$. aeruginosa, S. aureus and potentially pathogenic species emerging in CF such as Stenotrophomonas maltophilia, Achromobacter xylosoxidans and Serratia marcescens, in a large cohort of adults and children with CF monitored for 1 year.

\section{Materials and methods}

Patients

Fifty-nine patients followed at the Cystic Fibrosis Centre of Verona (Italy) were recruited in this study. Informed consent was obtained according to projects CRCFC-CEPPO026 and CRCFC-CEPPO031 approved by the local ethical committee. From all patients, we collected nasal lavages and sputum samples; from 38 of them, we also recovered saliva samples and used (2-3 months) toothbrushes. Patients were recruited at different times during the study, and samples were collected at routine visits (approximately every 3 months) from the moment of enrolment until the end of the collection period (fixed date for all patients). During the collection period, sputum, and nasal lavage were sampled at least twice from each patient. Collection of saliva and toothbrushes started at a later moment, and at least one sample of saliva and one toothbrush were collected from each patient. Patients were classified by age (adult and paediatric) and by stage of lung colonisation with $P$. aeruginosa at enrolment (chronic, occasional). Infection was defined as chronic with at least three positive cultures over $\geqslant 6$ months with at least a 1-month interval between the samples [13]. Based on these criteria, patients were divided into four groups: adults with chronic $P$. aeruginosa lung infection (AC), paediatrics with occasional $P$. aeruginosa colonisation (PO), adults and paediatrics free from $P$. aeruginosa (AN and $\mathrm{PN}$, respectively).

\section{Isolation and identification of bacterial strains}

Nasal lavage was performed as described by MaINZ et al. [12] by inserting $10 \mathrm{~mL}$ of sterile isotonic saline into each nostril [14]. The saliva was recovered by the unstimulated method, spitting in a sterile tube, 
while the sputum was collected by natural expectoration or by tracheal cannula (when the patient was unable to expectorate). The head of the toothbrush was detached and underwent five cycles of sonication (30 s each) in $5 \mathrm{~mL}$ of brain heart infusion medium before incubation for $24 \mathrm{~h}$ at $37^{\circ} \mathrm{C}$ with shaking. Saliva and sputum samples were treated with $0.5-1 \mathrm{~mL}$ of Sputolysin (Calbiochem, California, USA) and incubated for $40 \mathrm{~min}$ at $37^{\circ} \mathrm{C}$ with shaking. An aliquot of each sample was plated on Columbia blood (5\% sheep blood), McConkey, and Mannitol Salt agar plates (BD Difco, USA) and incubated at $37^{\circ} \mathrm{C}$ for $48 \mathrm{~h}$. All plates were kept at room temperature for an additional 5 days to recover small variant colonies and slow-growing strains. Isolated colonies were identified using MALDI-TOF-MS [15] and/or Vitek2 (Biomerieux, USA). Isolates were stored in Microbanks (ProLab Diagnostics, USA) at $-80^{\circ} \mathrm{C}$.

\section{Pulsed-field gel electrophoresis}

Suspensions were prepared from individual colonies after culture on Blood Columbia agar. Optical density at $600 \mathrm{~nm}$ was measured and suspensions were diluted to $10^{9} \mathrm{CFU} \cdot \mathrm{mL}^{-1}$ in EDTA-saline buffer $\left(75 \mathrm{mmol} \cdot \mathrm{L}^{-1} \mathrm{NaCl}\right.$ and $25 \mathrm{mmol} \cdot \mathrm{L}^{-1}$ EDTA, $\mathrm{pH}$ 7.5), then mixed with an equal volume of $1 \%$ low-melting-point agarose and allowed to solidify in a $100-\mu \mathrm{L}$ plug mould. The agarose plug was incubated for $24 \mathrm{~h}$ at $37^{\circ} \mathrm{C}$ in $500 \mu \mathrm{L}$ of lysis buffer $\left(6 \mathrm{mmol} \cdot \mathrm{L}^{-1}\right.$ Tris- $\mathrm{HCl}(\mathrm{pH} 7.6), 0.1 \mathrm{~mol} \cdot \mathrm{L}^{-1}$ EDTA, $1 \mathrm{~mol} \cdot \mathrm{L}^{-1} \mathrm{NaCl}, 0.5 \%$ Brij 58 (polyoxyethylene (20) cetyl ether; Sigma, USA)), 0.4\% sodium deoxycholate, $0.5 \%$ sodium lauryl sarcosine and $1 \mathrm{mg} \cdot \mathrm{mL}^{-1}$ lysozyme (and $10 \mu \mathrm{g} \cdot \mathrm{mL}^{-1}$ lysostaphin to S. aureus). The lysis buffer was replaced with $500 \mu \mathrm{L}$ of proteinase $\mathrm{K}$ buffer $\left(1 \%\right.$ sodium lauryl sarcosine, $0.5 \mathrm{~mol} \cdot \mathrm{L}^{-1}$ EDTA ( $\mathrm{pH} 9)$ and proteinase $\mathrm{K}\left(50 \mu \mathrm{g} \cdot \mathrm{mL}^{-1}\right.$; Sigma, USA)) and this solution was incubated with gentle shaking at $50^{\circ} \mathrm{C}$ for $20 \mathrm{~h}$. The plugs were then washed four times for $30 \mathrm{~min}$ at $37^{\circ} \mathrm{C}$ with $10 \mathrm{~mL}$ of Tris-EDTA buffer $\left(10 \mathrm{mmol} \cdot \mathrm{L}^{-1}\right.$ Tris- $\mathrm{HCl}(\mathrm{pH} 8)$ and $1 \mathrm{mmol} \cdot \mathrm{L}^{-1}$ EDTA). Half a slice of each plug was cut and incubated for 18-20 h with $30 \mathrm{U}$ of SpeI, or SmaI, XbaI, and Cfr9I in the restriction buffer (Thermo Fisher Scientific, USA). DNA restriction fragments were separated in a pulsed-field gel electrophoresis (PFGE) apparatus at $14^{\circ} \mathrm{C}, 6 \mathrm{~V} \cdot \mathrm{cm}^{-1}$, for a specific time for each species $(19-23 \mathrm{~h})$. The gel was stained with gel-red and visualised with a ultraviolet system [16].

\section{Genotype analysis}

DNA profiles were analysed with InfoQuest FP 5.1 (Bio-Rad Laboratories, Hercules, CA, USA), using the Dice correlation coefficient and unweighted pair group method with arithmetic mean (position tolerance and optimisation between 1.0 and 1.1\%). By applying the criteria of TeNOver et al. [17], based on the differences in the numbers of bands, we identified a cut-off value of $80 \%$ similarity to correctly cluster the PFGE profiles. Consequently, the strains showing $\geqslant 80 \%$ similarity were considered to be a single clone.

\section{Results}

Fifty-nine CF patients were enrolled and classified into four groups on the basis of their age (adult or paediatric) and the state of their lung colonisation by $P$. aeruginosa, as reported in table 1 . Two to five longitudinal samples were collected from each subject: from the majority of patients we recovered three samples of sputum $(n=44$ patients) and nasal lavage $(n=43)$, two samples of saliva $(n=28)$ and two toothbrushes $(n=22)$. Only in a few cases, a single saliva $(n=5)$ and toothbrush $(n=4)$ were collected (table 2). At the end of the study we had obtained and analysed 189 samples of sputum, 188 nasal lavages, 78 saliva samples, and 87 toothbrushes, for a total of 542 samples. From all these samples we isolated about 1200 bacterial strains, 530 of which belonging to the selected species: P. aeruginosa, S. maltophilia, A. xylosoxidans, S. marcescens, and S. aureus. At least four strains from each patient were genotyped by PFGE.

\begin{tabular}{|c|c|c|c|c|}
\hline \multirow[b]{2}{*}{ Group } & \multicolumn{4}{|c|}{ Study patients } \\
\hline & $A C$ & AN & PN & PO \\
\hline Number of patients & 9 & 15 & 25 & 10 \\
\hline Age years & $21(18-24)$ & $22.5(18-27)$ & $12.5(7-<18)$ & $12.5(7-<18)$ \\
\hline Male & $3(33.3 \%)$ & $10(66.7 \%)$ & $9(36 \%)$ & $4(40 \%)$ \\
\hline Female & $6(66.7 \%)$ & $5(33.3 \%)$ & $16(64 \%)$ & $6(60 \%)$ \\
\hline
\end{tabular}




\begin{tabular}{|c|c|c|c|c|c|}
\hline Type of sample & 1 sample & 2 samples & 3 samples & 4 samples & 5 samples \\
\hline Nasal lavage & 0 & 4 & 43 & 9 & 3 \\
\hline Sputum & 0 & 3 & 44 & 9 & 3 \\
\hline Saliva & 5 & 28 & 4 & 1 & 0 \\
\hline Toothbrush & 4 & 22 & 10 & 2 & 0 \\
\hline
\end{tabular}

\section{Bacterial presence in samples from adult patients}

All the AC individuals ( $\mathrm{n}=9$ ) showed presence of $P$. aeruginosa in sputum and nasal lavage. Only in one of these patients, in addition to $P$. aeruginosa we isolated $S$. aureus from both samples, whereas in all the other cases no other bacterial species were detected.

Regarding the AN group ( $n=15)$, the selected bacterial species were isolated from the nasal lavage and the sputum of 11 individuals (table 3). Seven patients (AN3, AN7, AN8, AN9, AN12, AN13, AN15) presented $S$. aureus in most of the samples. Interestingly, one of these patients (AN9), free from $P$. aeruginosa at the time of enrolment, acquired this bacterial species in both LAW and UAW at the first sampling. As regards the other investigated species, two patients (AN12 and AN15) resulted positive for S. aureus in all samples. Five patients were positive for A. xylosoxidans: AN2, AN4, AN10 in both sputum and nasal lavage, while AN5 and AN9 were only positive in the sputum. Within these five patients, except for AN4, the others were positive for the presence of $A$. xylosoxidans also in saliva. Another patient (AN14) was positive for S. maltophilia in sputum, nasal lavage and saliva samples obtained during four visits.

\section{Bacterial presence in samples from paediatric patients}

Within the PN group $(n=25)$, a number of individuals $(n=6)$ presented polyposis in their nasal/paranasal area. Although this is a condition that could favour the persistence of microbial cells, no difference in the prevalence of the investigated bacterial species was noticed between children with and without polyposis. Nineteen PN patients presented S. aureus in most nasal lavages and sputum samples. Thirteen of them were also positive for this species in saliva, and four also in the toothbrush. Among the people positive for S. aureus: 1) three children were positive also for S. maltophilia in most samples: PN4 (three sputum and two saliva samples), PN6 (three sputum samples) and PN9 (two nasal lavage, four sputum, and two saliva samples); 2) four patients were positive also for A. xylosoxidans in different types of samples: PN3 (three nasal lavage, four sputum, and three saliva samples), PN5 (one nasal lavage and two sputum samples), PN14 (one nasal lavage, three sputum and two saliva samples), PN17 (one sputum, two saliva samples and one toothbrush); 3) PN17 was positive also for P. aeruginosa in almost all the samples during four visits. Interestingly, patients PN1, PN12, and PN22 were initially positive for P. aeruginosa/S. aureus only in the nasal lavage, then these species were isolated also from sputum at a later visit (table 4).

Regarding the PO group ( $\mathrm{n}=10)$, seven children showed positive samples for the selected bacterial species, mainly S. aureus and P. aeruginosa. Interestingly, three (PO1, PO5, and PO8) presented the same bacterial species ( $S$. aureus alone or in combination with $P$. aeruginosa) in all types of samples. Moreover, PO6 presented Pseudomonas aeruginosa initially only in the nasal lavage but at the third visit this was also isolated from the sputum (table 5).

\section{Prevalence of the different species}

Considering as positive all the samples with at least one of the species investigated, in AN, PN, and PO patients the percentages of positivity were $87 \%$ for sputum, $66 \%$ for nasal lavage, $80 \%$ for saliva, and $30 \%$ for toothbrushes. We observed that $S$. aureus was the most abundant species in sputum (59\%), nasal lavage (48\%), saliva (56\%), and toothbrush (17\%), outdistancing the prevalence of the other investigated species. S. maltophilia and A. xylosoxidans were frequently present in LAW and oral cavity, being respectively isolated from $22 \%$ to $19 \%$ of sputum samples, and from $19 \%$ to $18 \%$ of saliva samples. Moreover, we detected them in $6 \%(n=10)$ and $7 \%(n=12)$ of nasal lavages, and in $3 \%(n=3)$ and $2 \%(n=2)$ of toothbrushes, respectively. P. aeruginosa showed high prevalence in sputum (16\%), nasal lavage (10\%), and saliva (10\%). S. marcescens was present in $6 \%$ of toothbrushes and never in nasal lavage (figure 1). The number and percentage of patients positive for each species in each type of sample are also reported in figure S1.

\section{Molecular typing}

The isolated bacterial strains were subjected to PFGE in order to obtain the genomic profile of every strain isolated from the different samples withdrawn from each patient. Figure 2 shows some examples regarding 
TABLE 3 Examined bacterial species collected from nasal lavage, sputum, saliva and toothbrush of adults free from Pseudomonas aeruginosa (AN) lung colonisation at different visits during the study

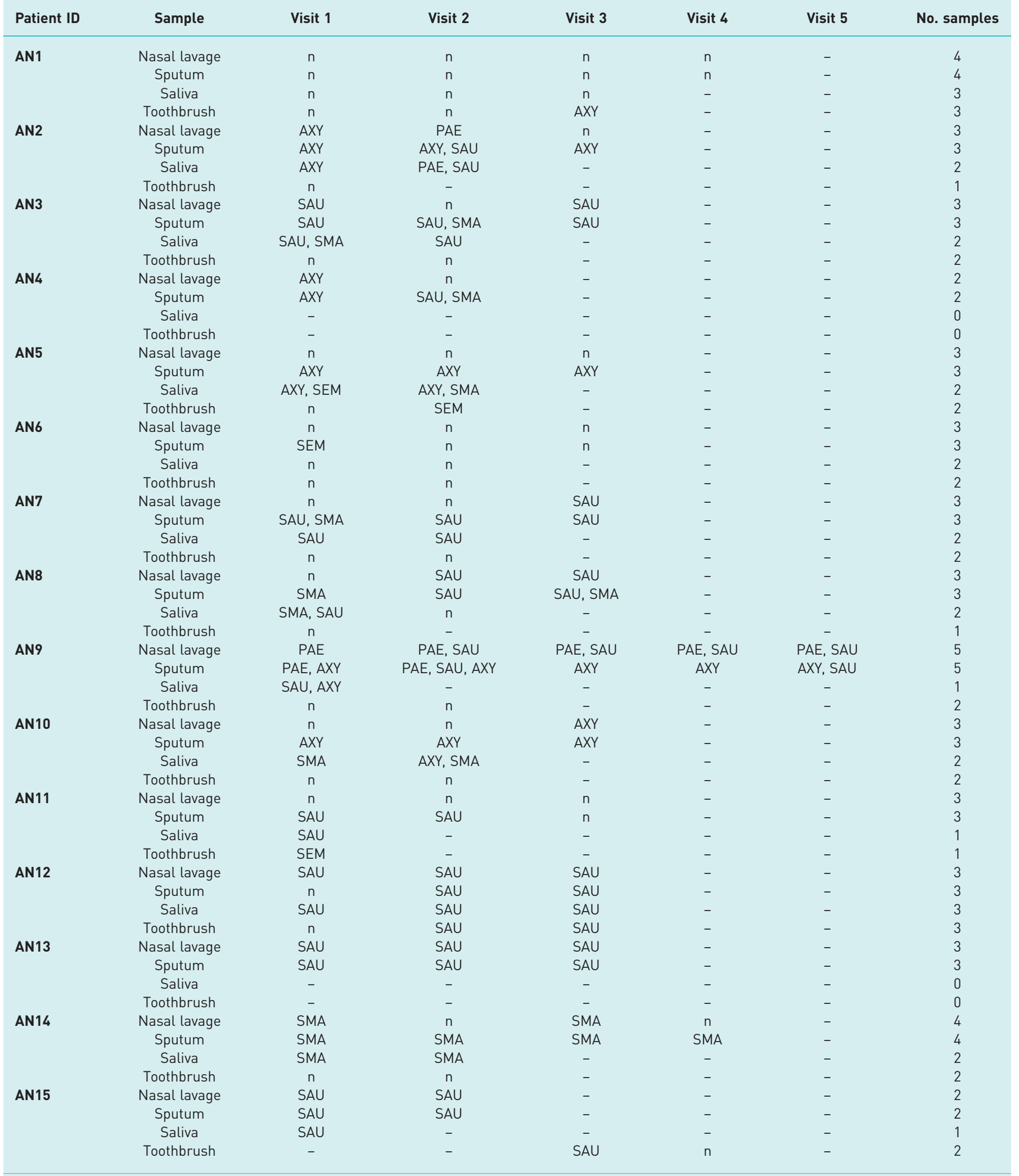

PAE: Pseudomonas aeruginosa; SAU: Staphylococcus aureus; SMA: Stenotrophomonas maltophilia; AXY: Achromobacter xylosoxidans; SEM: Serratia marcescens; n: no isolation of the investigated species; -: sample not available. Number of samples for each patient is also indicated. Patients with no saliva samples nor toothbrushes were not included in the collection of these samples. 
TABLE 4 Examined bacterial species collected from nasal lavage, sputum, saliva and toothbrush of paediatrics who had never been colonised by Pseudomonas aeruginosa (PN) at different visits during the study

\begin{tabular}{|c|c|c|c|c|c|c|c|}
\hline Patient ID & Sample & Visit 1 & Visit 2 & Visit 3 & Visit 4 & Visit 5 & No. samples \\
\hline \multirow{2}{*}{ PN1 } & Sputum & SAU & SAU, PAE & SAU & - & - & 3 \\
\hline & Toothbrush & $\mathrm{n}$ & $\mathrm{n}$ & - & - & - & 2 \\
\hline \multirow[t]{3}{*}{ PN2 } & Nasal lavage & SAU & SAU & SAU & - & - & 3 \\
\hline & Sputum & SAU & SAU & SAU, PAE & - & - & 3 \\
\hline & Saliva & SAU & SAU & - & - & - & 2 \\
\hline \multirow{3}{*}{ PN3 } & Sputum & AXY & AXY, SAU & AXY, SAU & AXY, SAU & - & 4 \\
\hline & Saliva & $A X Y$ & $A X Y$ & $A X Y$ & n & - & 4 \\
\hline & Toothbrush & $\mathrm{n}$ & $\mathrm{n}$ & $\mathrm{n}$ & $\mathrm{n}$ & - & 4 \\
\hline \multirow[t]{3}{*}{ PN4 } & Nasal lavage & $\mathrm{n}$ & SAU & SAU & - & - & 3 \\
\hline & Sputum & SAU, SMA, SEM & SAU, SMA & SAU, SMA & - & - & 3 \\
\hline & Saliva & SAU, SMA & SAU, SMA & - & - & - & 2 \\
\hline PN5 & Toothbrush & - & - & - & - & - & 0 \\
\hline \multirow[t]{4}{*}{ PN6 } & Nasal lavage & SAU & SAU & SAU & - & - & 3 \\
\hline & Sputum & SAU, SMA & SAU, SMA & SAU, SMA & - & - & 3 \\
\hline & Saliva & - & - & - & - & - & 0 \\
\hline & Toothbrush & - & - & - & - & - & 0 \\
\hline \multirow[t]{4}{*}{ PN7 } & Nasal lavage & $\mathrm{n}$ & SAU & $\mathrm{n}$ & - & - & 3 \\
\hline & Sputum & SAU & SAU & SAU, PAE & - & - & 3 \\
\hline & Saliva & SAU & SAU & - & - & - & 2 \\
\hline & Toothbrush & $\mathrm{n}$ & $\mathrm{n}$ & - & - & - & 2 \\
\hline \multirow[t]{3}{*}{ PN8 } & Nasal lavage & SAU & SAU & SAU & - & - & 3 \\
\hline & Sputum & SAU, SMA & SAU, SMA & SAU, PAE & - & - & 3 \\
\hline & Saliva & SAU, SMA & PAE & - & - & - & 2 \\
\hline PN10 & Toothbrush & - & - & - & - & - & 0 \\
\hline \multirow[t]{4}{*}{ PN11 } & Nasal lavage & SAU & SAU & SAU & - & - & 3 \\
\hline & Sputum & SAU & SAU & SAU, PAE & - & - & 3 \\
\hline & Saliva & SAU & - & - & - & - & 1 \\
\hline & Toothbrush & $\mathrm{n}$ & - & - & - & - & 1 \\
\hline \multirow[t]{4}{*}{ PN12 } & Nasal lavage & SAU & SAU & SAU, AXY & SAU & SAU & 5 \\
\hline & Sputum & SAU & SAU & $\mathrm{n}$ & $\mathrm{n}$ & SAU & 5 \\
\hline & Saliva & SAU & SAU & - & - & - & 2 \\
\hline & Toothbrush & SAU & $\mathrm{n}$ & - & - & - & 2 \\
\hline \multirow[t]{4}{*}{ PN13 } & Nasal lavage & SAU & SAU, SMA & SAU & - & - & 3 \\
\hline & Sputum & SAU & SAU, SMA & SAU & - & - & 3 \\
\hline & Saliva & - & - & - & - & - & 0 \\
\hline & Toothbrush & - & - & - & - & - & 0 \\
\hline \multirow[t]{4}{*}{ PN14 } & Nasal lavage & SAU, SMA & $\mathrm{n}$ & AXY, SMA & - & - & 3 \\
\hline & Sputum & SAU, AXY & SAU, AXY & SAU, AXY, SMA & - & - & 3 \\
\hline & Saliva & SAU, AXY & SAU, AXY & - & - & - & 2 \\
\hline & Toothbrush & $n$ & $n$ & - & - & - & 2 \\
\hline \multirow[t]{4}{*}{ PN15 } & Nasal lavage & $n$ & SAU & SAU & - & - & 3 \\
\hline & Sputum & $\mathrm{n}$ & SAU, AXY & SAU & - & - & 3 \\
\hline & Saliva & - & PAE & SAU & - & - & 2 \\
\hline & Toothbrush & $\mathrm{n}$ & $\mathrm{n}$ & PAE & - & - & 3 \\
\hline
\end{tabular}


TABLE 4 Continued

\begin{tabular}{|c|c|c|c|c|c|c|c|}
\hline Patient ID & Sample & Visit 1 & Visit 2 & Visit 3 & Visit 4 & Visit 5 & No. samples \\
\hline \multirow[t]{3}{*}{ PN16 } & Nasal lavage & $\mathrm{n}$ & $\mathrm{n}$ & SMA & - & - & 3 \\
\hline & Sputum & $\mathrm{n}$ & $n$ & SMA & - & - & 3 \\
\hline & Toothbrush & $\mathrm{n}$ & $n$ & - & - & - & 2 \\
\hline \multirow[t]{2}{*}{ PN17 } & Nasal lavage & SMA & SAU, PAE & SAU & SAU & - & 4 \\
\hline & Sputum & SAU, PAE, AXY & SAU, PAE & SAU, PAE & SAU, PAE & - & 4 \\
\hline \multirow[t]{4}{*}{ PN18 } & Nasal lavage & SAU & SAU & SAU & - & - & 3 \\
\hline & Sputum & SAU & SAU & SAU, SMA & - & - & 3 \\
\hline & Saliva & SAU & SAU & - & - & - & 2 \\
\hline & Toothbrush & $\mathrm{n}$ & $n$ & $n$ & - & - & 3 \\
\hline \multirow[t]{2}{*}{ PN19 } & Nasal lavage & $\mathrm{n}$ & $n$ & $n$ & $n$ & - & 4 \\
\hline & Sputum & SAU & SMA & $\mathrm{n}$ & $\mathrm{n}$ & - & 4 \\
\hline \multirow{2}{*}{ PN20 } & Saliva & SMA & $n$ & - & - & - & 2 \\
\hline & Toothbrush & SMA & $n$ & - & - & - & 2 \\
\hline \multirow[t]{4}{*}{ PN21 } & Nasal lavage & SAU & SAU & SAU & - & - & 3 \\
\hline & Sputum & SAU & SAU & SAU & - & - & 3 \\
\hline & Saliva & SAU & SAU & - & - & - & 2 \\
\hline & Toothbrush & SAU & SAU, SMA & SAU & - & - & 3 \\
\hline \multirow[t]{4}{*}{ PN22 } & Nasal lavage & SAU & SAU & SAU & - & - & 3 \\
\hline & Sputum & $\mathrm{n}$ & $\mathrm{n}$ & SAU & - & - & 3 \\
\hline & Saliva & SEM & $n$ & - & - & - & 2 \\
\hline & Toothbrush & $\mathrm{n}$ & $n$ & $\mathrm{n}$ & - & - & 3 \\
\hline \multirow[t]{3}{*}{ PN23 } & Nasal lavage & $n$ & SAU & SAU & PAE & - & 4 \\
\hline & Sputum & $n$ & SMA & SMA & SMA, PAE & - & 4 \\
\hline & Saliva & - & - & - & - & - & 0 \\
\hline PN25 & Toothbrush & $\mathrm{n}$ & $\mathrm{n}$ & $\mathrm{n}$ & - & - & 3 \\
\hline
\end{tabular}

PAE: Pseudomonas aeruginosa; SAU: Staphylococcus aureus; SMA: Stenotrophomonas maltophilia; AXY: Achromobacter xylosoxidans; SEM: Serratia marcescens; $n$ : no isolation of the investigated species; -: sample not available. Number of samples for each patient is also indicated. Patients with no saliva samples nor toothbrushes were not included in the collection of these samples.

the five bacterial species analysed and demonstrating the high level of similarity $(\geqslant 80 \%)$ existing among all the strains isolated from a single patient. For instance, $P$. aeruginosa strains isolated from patients AN9, PN1, PN17, and PO6 (figure 2a) show a $\geqslant 95 \%$ similarity between the different sites of isolation. Also, S. aureus strains collected from patients PN8, PN17, and PO8 (figure $2 b$ ) show a $\geqslant 97 \%$ similarity. High similarity is also shown within S. maltophilia ( $\geqslant 87 \%$, figure 2 c), S. marcescens $(\sim 92 \%$, figure $2 \mathrm{~d})$, and $A$. xylosoxidans $(\sim 95 \%$, figure 2e) strains. Among all the isolates examined, we identified only three cases showing $<80 \%$ similarity between strains isolated at the different visits (AC4, AC9, and PN12).

The PFGE profiles of representative strains isolated from the sputum of each patient were compared to identify clones that might be shared among patients. Clonal S. aureus strains were detected in three pairs of individuals (AN11 and AN9, PN7 and PN12, PN1 and AN7), as shown in figure 3 (indicated by braces).

\section{Discussion}

One of the most important challenges in CF is treating and solving chronic lung infections, which are the major cause of morbidity and mortality in patients. The adaptation of some bacterial species such as 
TABLE 5 Examined bacterial species collected from nasal lavage, sputum, saliva and toothbrush of paediatrics occasionally colonised by Pseudomonas aeruginosa (PO) at different visits during the study

\begin{tabular}{|c|c|c|c|c|c|c|c|}
\hline Patient ID & Sample & Visit 1 & Visit 2 & Visit 3 & Visit 4 & Visit 5 & No. samples \\
\hline \multirow{2}{*}{ P01 } & Sputum & PAE & SAU & SAU, PAE & SAU, PAE & - & 4 \\
\hline & Toothbrush & $\mathrm{n}$ & PAE & PAE & - & - & 3 \\
\hline \multirow[t]{2}{*}{ P02 } & Nasal lavage & $\mathrm{n}$ & $\mathrm{n}$ & SAU & - & - & 3 \\
\hline & Sputum & $\mathrm{n}$ & SAU & $\mathrm{n}$ & - & - & 3 \\
\hline \multirow[t]{4}{*}{ P03 } & Nasal lavage & $\mathrm{n}$ & $n$ & - & - & - & 2 \\
\hline & Sputum & PAE & PAE & & - & - & 2 \\
\hline & Saliva & $\mathrm{n}$ & $\mathrm{n}$ & - & - & - & 2 \\
\hline & Toothbrush & $n$ & $n$ & - & - & - & 2 \\
\hline \multirow[t]{2}{*}{ P04 } & Nasal lavage & $\mathrm{n}$ & $\mathrm{n}$ & $n$ & - & - & 3 \\
\hline & Sputum & SAU & SAU, SMA & SAU, SEM & - & - & 3 \\
\hline P05 & Toothbrush & SAU, PAE & $\mathrm{n}$ & - & - & - & 2 \\
\hline \multirow[t]{4}{*}{ P06 } & Nasal lavage & PAE & $\mathrm{n}$ & $\mathrm{n}$ & PAE & - & 4 \\
\hline & Sputum & $\mathrm{n}$ & $\mathrm{n}$ & PAE & $\mathrm{n}$ & - & 4 \\
\hline & Saliva & - & - & - & - & - & 0 \\
\hline & Toothbrush & - & - & - & - & - & 0 \\
\hline \multirow[t]{4}{*}{ P07 } & Nasal lavage & PAE & $n$ & $n$ & - & - & 3 \\
\hline & Sputum & PAE, SEM, SMA & PAE, SEM & PAE, SEM & - & - & 3 \\
\hline & Saliva & - & - & - & - & - & 0 \\
\hline & Toothbrush & - & - & - & - & - & 0 \\
\hline \multirow[t]{3}{*}{ P08 } & Nasal lavage & $\mathrm{n}$ & SAU & $\mathrm{n}$ & SAU & SAU & 5 \\
\hline & Sputum & SAU & SAU, SEM & SAU & SAU, SMA & SAU & 5 \\
\hline & Saliva & SAU, SMA & SAU & SAU & - & - & 3 \\
\hline P010 & Toothbrush & $\mathrm{n}$ & $\mathrm{n}$ & - & - & - & 2 \\
\hline
\end{tabular}

PAE: Pseudomonas aeruginosa; SAU: Staphylococcus aureus; SMA: Stenotrophomonas maltophilia; AXY: Achromobacter xylosoxidans; SEM: Serratia marcescens; n: no isolation of the investigated species; -: sample not available. Number of samples for each patient is also indicated. Patients with no saliva samples nor toothbrushes were not included in the collection of these samples.

$P$. aeruginosa to the lung environment is the most important mechanism of infection, allowing the bacteria to evolve from acute to chronic colonisation. However, a role for bacterial reservoirs located in other body districts connected to and feeding the lower airways has also been hypothesised [4, 7, 10, 12]. It seems clear that their presence constitutes a continuing risk of chronic lung [18] recontamination [19]. Lack of eradication of potentially pathogenic bacteria in those reservoirs could lead to a continuous source of infectious agents producing lung colonisation and finally chronic infection [19]. The nasal/paranasal sinuses have been proven to be a reservoir both in people with CF and primary ciliary dyskinesia. MorLaCCH et al. [20] demonstrated that a large number of patients had the same pathogens colonising both LAW and UAW after lung transplant. In addition, a number of studies have shown that P. aeruginosa and $S$. aureus clones can be found both in the LAW and UAW of these patients [12, 21]. Also, the oral cavity, as well as objects introduced in the mouth such as the toothbrush, were shown to have a role as bacterial reservoirs [11, 22]. As highlighted in a recent literature review [11], it is still not clear whether typical CF bacterial species are transient members of the oral flora in patients with occasional and chronic bronchopulmonary colonisation. The analysis of the specific literature performed in this review leads to 
FIGURE 1 Prevalence of the examined species isolated from the sputum, nasal lavage, saliva and toothbrush lconsidering adult negative, paediatric negative and paediatric occasional). Percentage of positive samples is indicated on the $\mathrm{x}$-axis; number of positive samples is shown above bars.

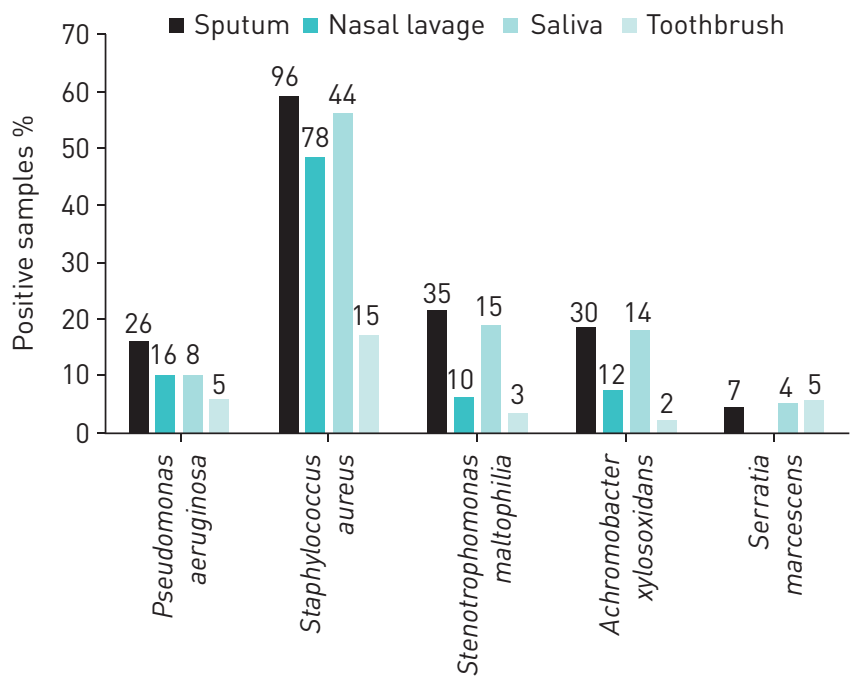

the conclusion that more longitudinal studies are needed in the CF population to learn about the prevalence of typical CF bacterial species and the genotypic concordance between specific strains present in reservoirs and causing lung colonisation. For these reasons, in this study we have considered not only the classical sputum sample but also nasal lavage, saliva, and toothbrushes, and have monitored patients longitudinally. Interestingly, previous studies showed that specific IgA against $P$. aeruginosa in serum, sputum, nasal lavage, and saliva can contribute to differentiation between patients chronically infected, intermittently colonised, and without $P$. aeruginosa in the lungs [23, 24]. However, their predictability about infection in other sites like UAW, as well as the possibility of cross-reaction with other Gram-negative pathogens need to be further clarified.

As patients were recruited at different times during the study, we collected different numbers of samples according to the time each patient was enrolled. For example, the first enrolled patients (PN12, PO8, AN9) produced up to five longitudinal samples of sputum and nasal lavage, whereas the last enrolled patients (PO3, AN4, AN15) produced only two samples. The collection of saliva and toothbrushes started later, thus for the majority of patients $(n=27)$ there are fewer oral than respiratory samples. Also, in few cases a sample was missed $(n=12)$ due to collection-related issues: patient PN10 missed the collection of nasal lavage at the second visit, while in all the other cases the missing sample was saliva $(n=9)$ or toothbrush $(n=2)$. As an only exception, we included in the study two toothbrushes that were collected from patient AN15 after the end of the collection period. Despite these deviations in sampling, we managed to collect at least two longitudinal samples of sputum and nasal lavage from all patients, and at least one sample of saliva and one toothbrush. This number of samples was sufficient to allow the comparison between the species and strains isolated from the four different districts.

Differently from previous works, our study was not limited to $P$. aeruginosa and $S$. aureus (the most important CF pathogens, responsible for the majority of lung infections and for the decrease of lung functionality) but also included other potentially pathogenic emerging bacterial species such as A. xylosoxidans, S. maltophilia, and S. marcescens, that are frequently isolated from the sputum of CF subjects but still lack a clear clinical role in CF $[25,26]$. While $S$. aureus and $P$. aeruginosa were present in the samples from $68 \%$ to $48 \%$ of our patients, respectively, we detected S. maltophilia and A. xylosoxidans in the respiratory samples of $39 \%(n=23)$ and $22 \%(n=13)$ of all 59 patients enrolled, respectively. Prevalence of each species in the different types of samples is summarised in table S2.

We have been monitoring adults and paediatrics who were never or only occasionally colonised by $P$. aeruginosa, as this is the population that might benefit more from monitoring of bacterial reservoirs to avoiding the insurgence of a chronic lung infection. In addition, a small number of adults with $P$. aeruginosa chronic lung colonisation were monitored as a control: in these patients, as expected, we isolated the same $P$. aeruginosa clones from all the investigated sites. About $80 \%$ of the 59 subjects monitored in this study resulted positive for at least one of the investigated bacterial species. Overall, $74 \%$ of the patients who resulted positive in the sputum also presented the same species in the nasal lavage and saliva. The interpretation of microbiota data is particularly challenging due to concerns about optimal sampling techniques and crossover between upper and lower airway bacterial communities [27]. Although we cannot exclude cross-contamination between sputum and saliva during the sampling, the repetitive isolation of the same bacterial strain in the different samples supports the persistence of bacteria also in 
a)

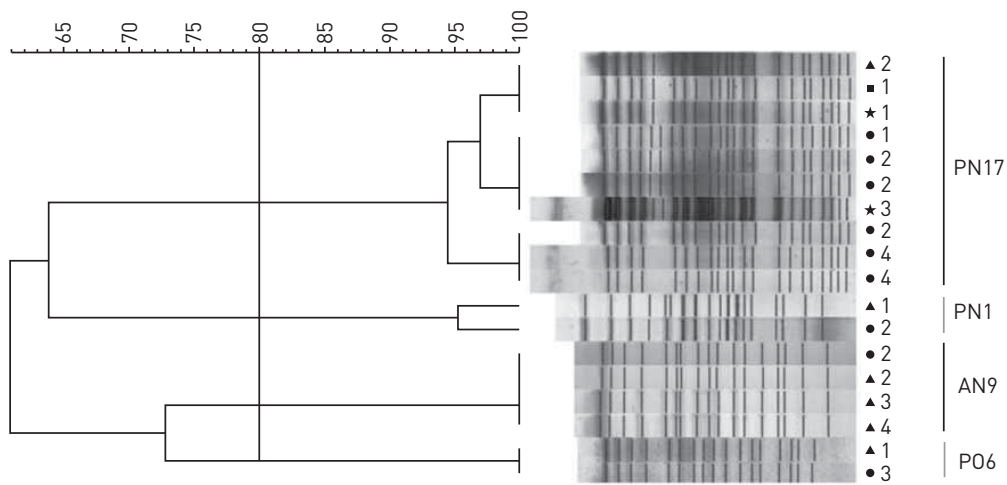

b)
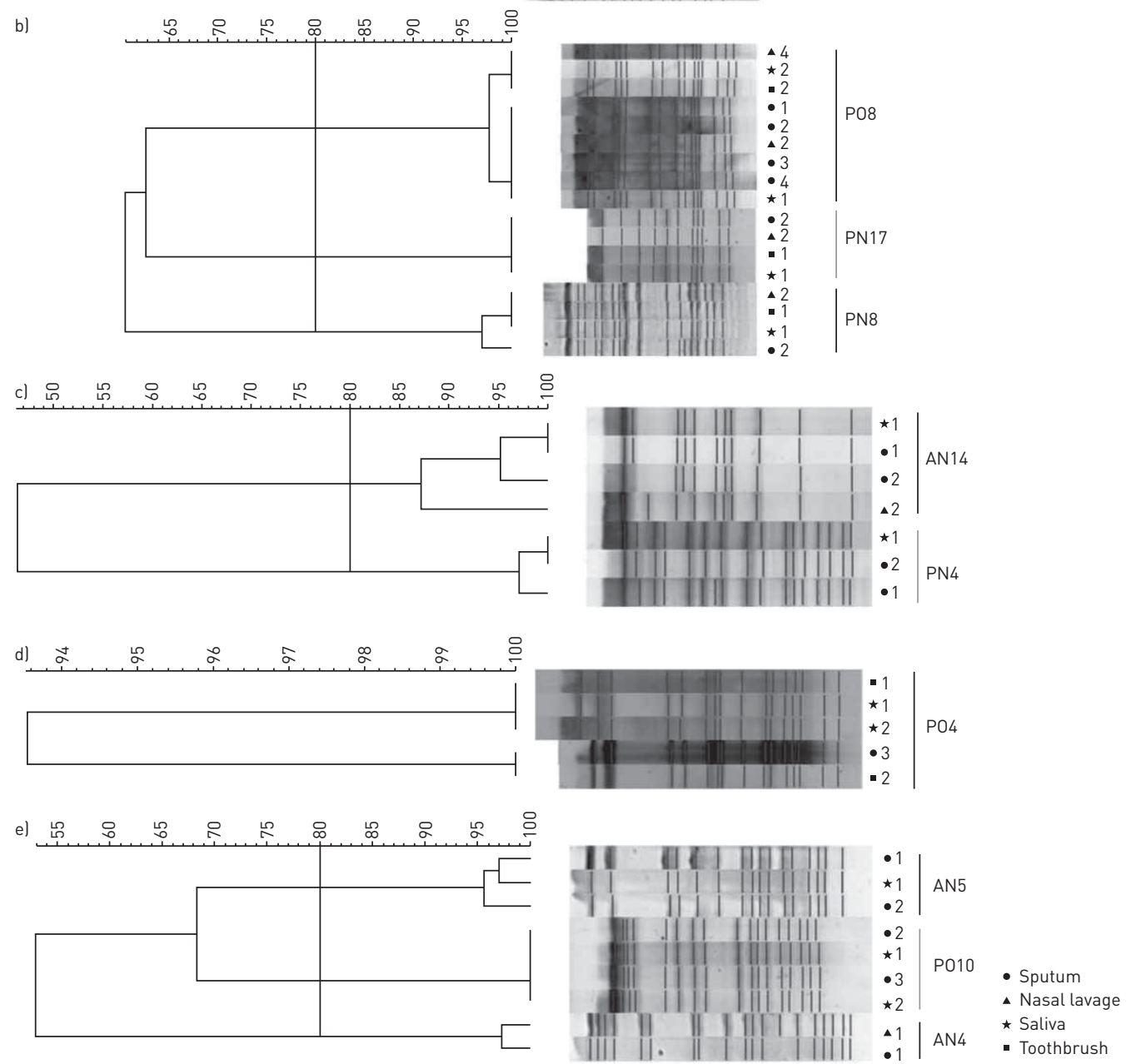

FIGURE 2 Phylogenetic trees obtained from the analysis of pulsed-field gel electrophoresis profiles of a) Pseudomonas aeruginosa, b) Staphylococcus aureus, c) Stenotrophomonas maltophilia, d) Serratia marcescens and e) Achromobacter xylosoxidans. Numbers indicate longitudinal visits. The threshold of $80 \%$ similarity is indicated by the vertical line. AN: adult negative; PN: paediatric negative; PO: paediatric occasional.

the oral cavity. Thus, we stress the importance of monitoring saliva as a read-out of the mouth microflora. As regards toothbrushes, $26 \%$ of the samples were positive for the same bacterial species found in sputum and, in some cases, also in the other two types of samples. Although this percentage is lower than that of the sinuses and the oral cavity, it is significant when considering a source of bacteria potentially associated with LAW infection. It therefore seems important to properly educate people with CF on how to maintain their toothbrush free of bacterial contamination.

The molecular typing of the bacterial strains isolated from the different samples of each patient indicates that most of them belong to single clones circulating in the UAW, LAW and oral cavity and, in some cases, in the toothbrush too. Regarding the possibility of transmission between the investigated reservoirs and the lungs, it is worth highlighting some interesting cases. Three children (PN1, PN22, PO6) initially 

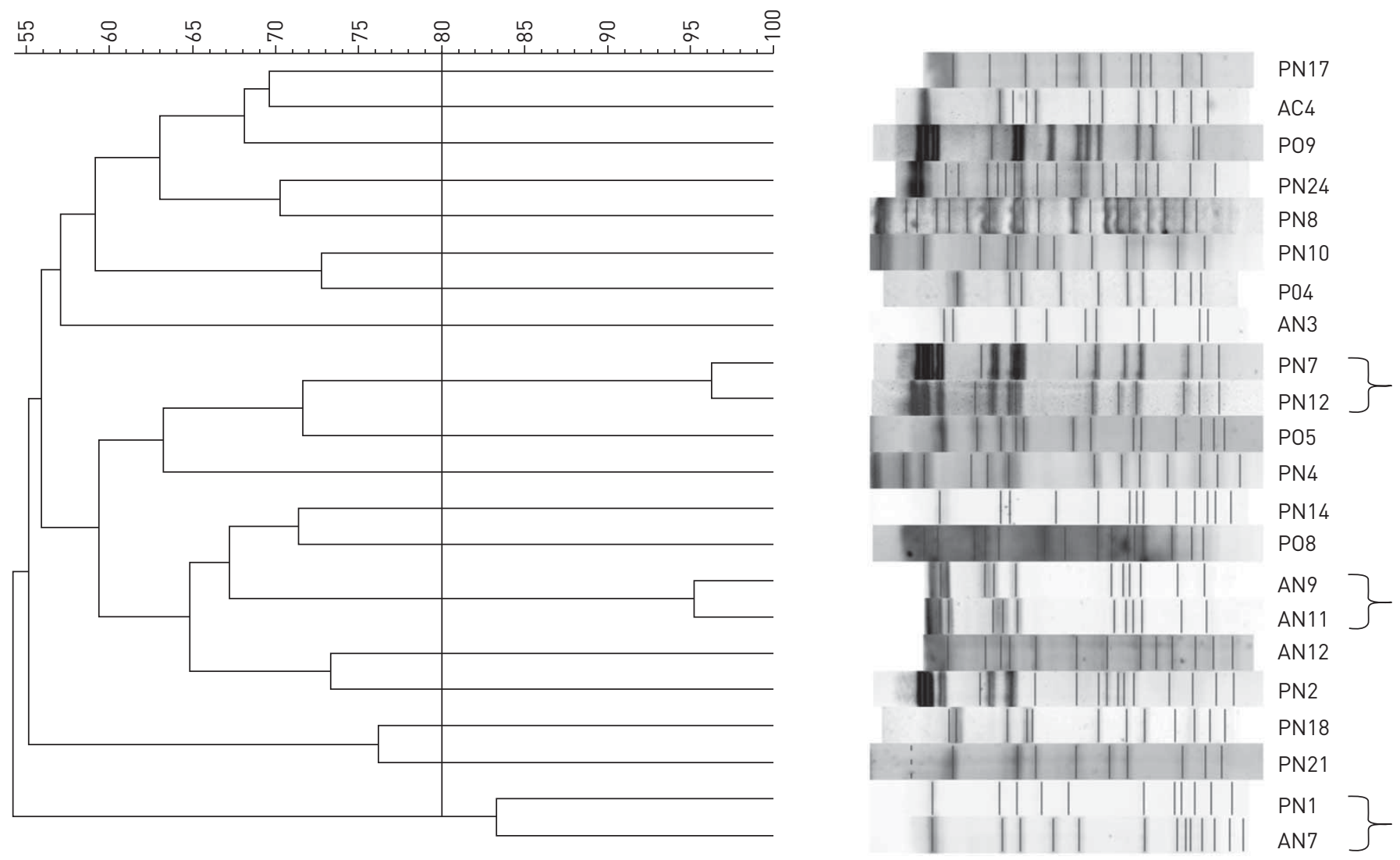

FIGURE 3 Phylogenetic trees obtained from the analysis of pulsed-field gel electrophoresis profiles of Staphylococcus aureus isolated from sputum samples. The threshold of $80 \%$ similarity is indicated by the vertical line. Braces indicate strains from different patients with $>80 \%$ similarity. AC: adult chronic; AN: adult negative; PN: paediatric negative; PO: paediatric occasional.

showing colonisation of the UAW became positive for the same clone also in the LAW by the end of the study. PN1 presented P. aeruginosa only in the nasal lavage at the first visit, while in the second visit the pathogen was detected in the sputum too. PN22 presented S. aureus only in the nasal lavage at the first two visits and also in the sputum at the third visit. At the first visit PO6 presented P. aeruginosa only in the nasal lavage, in the third visit only in the sputum and in the fourth visit only in the nasal lavage again; although $P$. aeruginosa was undetected at the second visit, LAW colonisation with the same clone support the possible passage from UAW. Similarly, in two patients (PO4, $\mathrm{PO} 9)$ the bacterial strains were present in the saliva, and the same clones were later isolated also from the sputum. An adult patient (AN9) was colonised at the beginning of the study by $P$. aeruginosa in both LAW and UAW; after combined intravenous antibiotic therapy with ceftazidime and azithromycin, $P$. aeruginosa was successfully eradicated only in the LAW. However, the infection persisted in the UAW and, after the end of the study, lung colonisation was detected again. This might indicate that the same microorganism, when persisting in the nasal sinuses, can transfer to the lungs, where it can cause a chronic infection. These cases highlight the likelihood of transmission of potential pathogens from nasal/oral reservoirs to the lung environment, and the importance of typical CF bacteria eradication from these reservoirs to prevent lung infections. While further evidence regarding the role of oral reservoir might be needed, our study confirms the need for eradication of bacterial CF species from the UAW in addition to standard LAW eradication.

\section{Conclusion}

We can conclude that nasal and oral sites act as bacterial reservoirs favouring the transmission of potentially pathogenic microorganisms to the lower airways. The lack of eradication from these sites might undermine the antibiotic therapy applied to treat the lung infection, allowing the persistence of the bacteria within the patient, in a continuous cycle of lung eradication and re-infection. Thus, it might be useful to introduce in the clinical routine of $\mathrm{CF}$ centres the monitoring of bacterial reservoirs, such as nasal sinuses and oral cavity, and the training of CF patients to properly clean and store toothbrushes, in order to prevent the establishment of lung infections due to infectious agents colonising and persisting in these sites. 
Acknowledgements: We thank Clizia Cazzarolli for her support in enrolling patients, Alessandra Grilli for collecting toothbrushes and Giovanna Amenta for the nursing service (Cystic Fibrosis Centre of Verona).

Conflict of interest: R. Passarelli Mantovani has nothing to disclose. A. Sandri has nothing to disclose. M. Boaretti has nothing to disclose. G. Burlacchini has nothing to disclose. V. Li Vigni has nothing to disclose. M. Scarazzai has nothing to disclose. P. Melotti has nothing to disclose. C. Signoretto reports grants from Fondazione per la Ricerca sulla Fibrosi Cistica during the conduct of the study. M.M. Lleo has nothing to disclose.

Support statement: This study was supported by the Italian Cystic Fibrosis Research Foundation (project FFC\#22/2016 adopted by Delegazione FFC di Treviso Montebelluna). Funding information for this article has been deposited with the Crossref Funder Registry.

\section{References}

1 Caverly LJ, LiPuma JJ. Cystic fibrosis respiratory microbiota: unraveling complexity to inform clinical practice. Expert Rev Respir Med 2018; 12: 857-865.

2 Oliver A. Mutators in cystic fibrosis chronic lung infection: prevalence, mechanisms, and consequences for antimicrobial therapy. Int J Med Microbiol 2010; 300: 563-572.

3 Moller ME, Alanin MC, Gronhoj C, et al. Sinus bacteriology in patients with cystic fibrosis or primary ciliary dyskinesia: a systematic review. Am J Rhinol Allergy 2017; 31: 293-298.

4 Aanaes K. Bacterial sinusitis can be a focus for initial lung colonisation and chronic lung infection in patients with cystic fibrosis. J Cyst Fibros 2013; 12: Suppl. 2, S1-20.

5 Berkhout MC, Klerx-Melis F, Fokkens WJ, et al. CT-abnormalities, bacteriology and symptoms of sinonasa disease in children with cystic fibrosis. J Cyst Fibros 2016; 15: 816-824.

6 Sakano E, Ribeiro AF, Barth L, et al. Nasal and paranasal sinus endoscopy, computed tomography and microbiology of upper airways and the correlations with genotype and severity of cystic fibrosis. Int J Pediatr Otorhinolaryngol 2007; 71: 41-50

7 Hansen SK, Rau MH, Johansen HK, et al. Evolution and diversification of Pseudomonas aeruginosa in the paranasal sinuses of cystic fibrosis children have implications for chronic lung infection. ISME J 2012; 6: 31-45

8 Walter S, Gudowius P, Bosshammer J, et al. Epidemiology of chronic Pseudomonas aeruginosa infections in the airways of lung transplant recipients with cystic fibrosis. Thorax 1997; 52: 318-321.

9 Fischer N, Hentschel J, Markert UR, et al. Non-invasive assessment of upper and lower airway infection and inflammation in CF patients. Pediatr Pulmonol 2014; 49: 1065-1075.

10 Mainz JG, Michl R, Pfister W, et al. Cystic fibrosis upper airways primary colonization with Pseudomonas aeruginosa: eradicated by sinonasal antibiotic inhalation. Am J Respir Crit Care Med 2011; 184: 1089-1090.

11 Rivas Caldas R, Boisrame S. Upper aero-digestive contamination by Pseudomonas aeruginosa and implications in cystic fibrosis. J Cyst Fibros 2015; 14: 6-15.

12 Mainz JG, Naehrlich L, Schien M, et al. Concordant genotype of upper and lower airways $P$. aeruginosa and S. aureus isolates in cystic fibrosis. Thorax 2009; 64: 535-540.

13 Doring G, Conway SP, Heijerman HG, et al. Antibiotic therapy against Pseudomonas aeruginosa in cystic fibrosis: a European consensus. Eur Respir J 2000; 16: 749-767.

14 Beiersdorf N, Schien M, Hentschel J, et al. Soluble inflammation markers in nasal lavage from CF patients and healthy controls. J Cyst Fibros 2013; 12: 249-257.

15 Desai AP, Stanley T, Atuan M, et al. Use of matrix assisted laser desorption ionisation-time of flight mass spectrometry in a paediatric clinical laboratory for identification of bacteria commonly isolated from cystic fibrosis patients. J Clin Pathol 2012; 65: 835-838.

16 Goering RV. Pulsed-field gel electrophoresis: a review of application and interpretation in the molecular epidemiology of infectious disease. Infect Genet Evol 2010; 10: 866-875.

17 Tenover FC, Arbeit RD, Goering RV, et al. Interpreting chromosomal DNA restriction patterns produced by pulsed-field gel electrophoresis: criteria for bacterial strain typing. J Clin Microbiol 1995; 33: 2233-2239.

18 Folkesson A, Jelsbak L, Yang L, et al. Adaptation of Pseudomonas aeruginosa to the cystic fibrosis airway: an evolutionary perspective. Nat Rev Microbiol 2012; 10: 841-851.

19 Aanaes K, Eickhardt S, Johansen HK, et al. Sinus biofilms in patients with cystic fibrosis: is adjusted eradication therapy needed? Eur Arch Otorhinolaryngol 2015; 272: 2291-2297.

20 Morlacchi LC, Greer M, Tudorache I, et al. The burden of sinus disease in cystic fibrosis lung transplant recipients. Transpl Infect Dis 2018; 20: e12924.

21 Alanin MC, Johansen HK, Aanaes K, et al. Simultaneous sinus and lung infections in patients with primary ciliary dyskinesia. Acta Otolaryngol 2015; 135: 58-63.

22 Linnane B, Collins L, Bussmann N, et al. Medical devices for cystic fibrosis care may be portable reservoirs of potential pathogens. J Hosp Infect 2017; 96: 397-398.

23 Aanaes K, Johansen HK, Poulsen SS, et al. Secretory IgA as a diagnostic tool for Pseudomonas aeruginosa respiratory colonization. J Cyst Fibros 2013; 12: 81-87.

24 Mauch RM, Hentschel J, Aanaes K, et al. Antibody response against Pseudomonas aeruginosa and its relationship with immune mediators in the upper and lower airways of cystic fibrosis patients. Pediatr Pulmonol 2020; 55 959-967.

25 Green H, Jones AM. Emerging Gram-negative bacteria: pathogenic or innocent bystanders. Curr Opin Pulm Med 2018; 24: 592-598.

26 Recio R, Branas P, Martinez MT, et al. Effect of respiratory Achromobacter spp. infection on pulmonary function in patients with cystic fibrosis. J Med Microbiol 2018; 67: 952-956.

27 Zemanick ET, Wagner BD, Robertson CE, et al. Assessment of airway microbiota and inflammation in cystic fibrosis using multiple sampling methods. Ann Am Thorac Soc 2015; 12: 221-229. 